\title{
Short-Term Crude Oil Scheduling Modeling and Algorithm with High Fusion Point Oil and Two Pipelines Transportation Based on Priority-Slot
}

\author{
Yuming Zhao ${ }^{1, \text { a * }}$ \\ ${ }^{1}$ School of computer Science, Zhaoqing University, Zhaoqing 526061, China \\ aymzhao@zqu.edu.cn
}

Keywords: Oil refinery, Continuous-time formulation, Two pipelines transfer, High-fusion-point oil transfer

\begin{abstract}
Some oil refineries arrange low fusion point crude oil storage tanks and high fusion point ones at two different locations. Different fusion point crude oil needs different pipeline to transfer. So, to transport diverse fusion point oil needs two pipelines. Because of the constraints resulted from transferring high fusion point oil, to schedule such an organization is hard. This paper proposes a new MINLP formulation with transferring high-fusion-point oil and two pipelines transfer that is based on single-operation sequencing (SOS). This formulation aims at minimizing the sum of high-fusion-point oil transfer cost. Simple two-stage MILP-NLP heuristic method cannot be applied to solve the model, so we give an iterative algorithm directly using MINLP solver DICOPT to solve it.
\end{abstract}

\section{Introduction}

A great challenge may occur during operating an oil refinery. Mixed integer programming-based techniques, especially mixed integer linear programming (MILP), have been preferred to solve crude oil scheduling problem [2]. Based on time grids, a mixed integer programming formulation can easily model the capacity of a tank or production unit at each time interval end [1].

Through mixed integer programming models, the uniform time discretization formulations have been successfully applied in solving batch processes representation based on an STN or RTN. For the sake of reducing the discrete variable number, non-uniform time discretization formulations have been set up on the basis of an RTN or STN representation [3]. This method is also to fulfill easily and is applicable for scheduling problems with a long horizon as it results in more abbreviated model.

In many refineries, every one of them locates the storage tanks at one site. First, refinery unloads crude oil of all the types into storage tanks, and then delivers them to the charging ones through only one pipeline. In this situation, schedulability constraints are got on the hypothesis that there is no processing of high fusion point oil, whose abbreviation is $\mathrm{H}$-oil [3]. When $\mathrm{H}$-oil needs processing, the $\mathrm{H}$-oil in storage tanks has to be delivered into charging tanks and setup cost of these delivery is very high, that farther makes the short-term scheduling problem complicated. Therefore, to constitute the schedulability constraints and determine how much crude oil can be transferred using an individual setup for the sake of minimizing the transfer cost becomes indispensable.

In many other situations, because of the particular prerequisite of $\mathrm{H}$-oil offloading from a vessel, the refinery locates the storage tanks at two geographically diverse locations rather than one. The storage tanks at diverse locations are employed to store low fusion point crude oil (named L-oil for short) and H-oil, respectively. Because of two locations of storage tanks, crude oil must be delivered from storage tanks to charging tanks using two pipelines. Before transferring $\mathrm{H}$-oil, the refinery must head the pipeline first. During hot L-oil in the charging tanks flowing through the pipeline into storage ones, it becomes hot enough such that H-oil is capable of flowing in it. After finishing the H-oil transfer, it becomes at leisure. Nonetheless H-oil cannot sojourn and keep immobile, because otherwise H-oil could be solidified so as to congest it. Therefore, one must exploit L-oil to run across it in the same orientation to eject H-oil totally. Evidently, the crude oil stream in it has bidirectional orientation. Thus, some charging tanks should not be employed in charging distillers but in 
transferring oil, which requires more charging tanks. In order to decrease operation cost, to transfer as much as possible $\mathrm{H}$-oil of type from storage tanks at a time to the refinery with an individual setup. As far as we've known, there is no research report to address such a refinery short-term scheduling using mathematical programming with the above-described pipelines.

Recently, Mouret et al. [4] propose a continuous time formulation based on priority-slot for the crude oil operation scheduling problem. The most special advantage of such a formulation is that the total operation number to be performed by the obtained schedule is the only parameter that needs to be known in advance. Tackling L-oil and H-oil with two pipelines results in different operations makes the scheduling problem much more challenging. This work aims at creating a formulation based on single-operation sequencing (SOS) which can decide the required $\mathrm{H}$-oil amount in storage tanks that can be delivered to charging ones, as well how to transfer it. The problem is formulated as a mixed integer programming with continuous-time representation by using the priority-slot-based modeling method.

\section{The scheduling problem with $\mathrm{H}-0 \mathrm{il}$ and two pipelines transfer}

Oil refinery processes usually involving three phases are illustrated: (1) crude oil operations; (2) production; and (3) product delivery. A variety of crude oil types need refining in a refinery which includes $\mathrm{H}$-oil. The fusion point of $\mathrm{H}$-oil usually is higher than $30^{\circ} \mathrm{C}$ and at normal temperature its state is solid. Usually, to facilitate unloading, storing, transferring crude oil through a submarine pipeline for crude oil vessels, a location is built. Location \#1 in Fig. 1 is exploited to illustrate such a location. Unfortunately, the submarine temperature is invariably under $30^{\circ} \mathrm{C}$ and such an undersea pipeline cannot permit $\mathrm{H}$-oil to transfer through it. Therefore, a refinery processing $\mathrm{H}$-oil have no choice but to utilize a jetty which is sufficiently close to the land such that a grounded can be constructed. This kind of jetty is usually small-scale and can only anchor small tankers. In Fig. 1, we apply Location \#2 to indicate this kind of facility. Using small tankers leads to the relatively high transfer cost, so L-oil is delivered to Location \#1 and only $\mathrm{H}$-oil is to Location \#2.

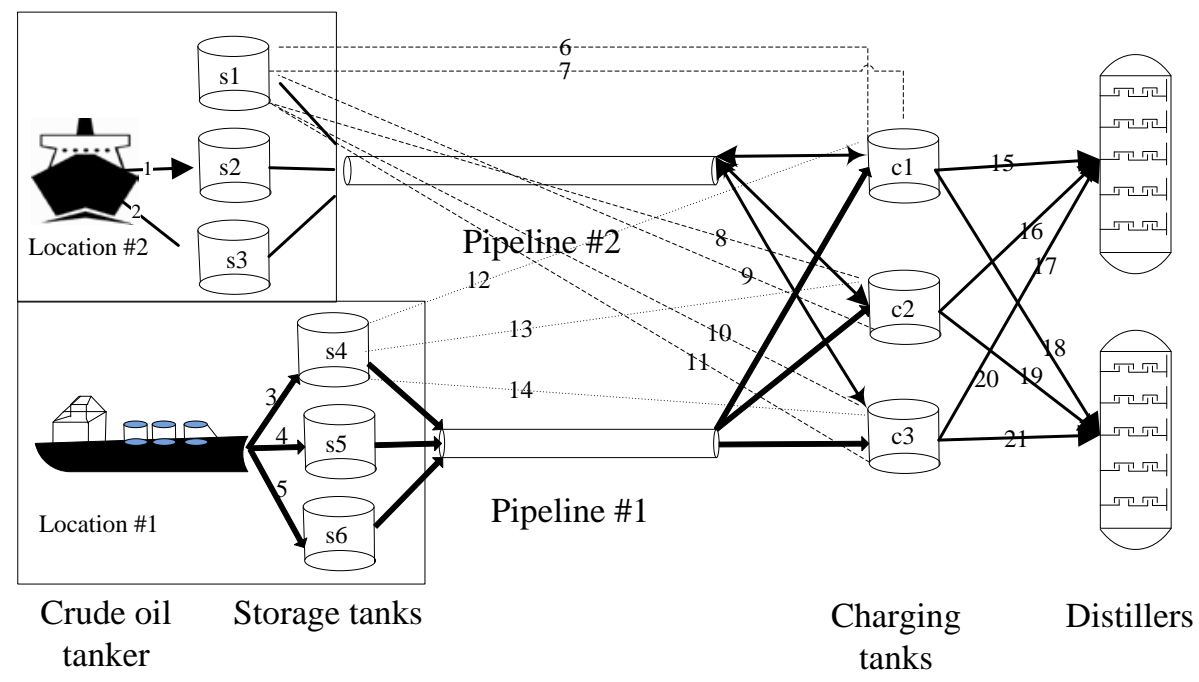

Figure 1. The refinery configuration for modeling illustration

Before Pipeline \#2 being charged it should be heated in case H-oil becomes frozen throughout the course of its transfer. Only the flowing of hot L-oil in charging tanks through Pipeline \#2 backwards to storage tanks at Location \#2 can heat it. When it is sufficiently hot, the hot H-oil can be transferred from storage tanks at Location \#2 where it is contained to charging tanks. When H-oil is discharged in Pipeline \#2, it has to be kept fluid; otherwise ambient temperature would make the pipeline cooling and the $\mathrm{H}$-oil within it would also be cooled so as to become solid. After the last $\mathrm{H}$-oil parcel needing transferring is emitted into the pipeline completely, it is necessary that to emit another L-oil parcel in the storage tanks at Location \#2 into the pipeline to eject the H-oil within it totally. It is required that Location \#2 should have sufficient L-oil and the refinery's side should possess adequate space of 
charging tank. There are novel questions which do not exist in one-pipeline systems, for example, under what circumstances and when to transfer H-oil, as well how to make the operations for both pipelines in concert with each other.

\section{Problem Formulation}

This section presents the priority-slot-based continuous-time formulation for the short-term crude oil operations scheduling problem with high-fusion-point oil and two pipelines transfer.

Sets and parameters: $T=\{1,2, \ldots, n\}:$ Set of priority-slots; $W$ :Set of the $n$ ODs for a schedule: $W=W_{U} \cup W_{T} \cup W_{D}\left(W=\{1-21\}\right.$ for the system shown in Fig. 1); $W_{U H}$ : Set of unloading ODs of H-oil vessels; $W_{U L}$ : Set of unloading ODs of L-oil vessels; $W_{U}=W_{U H} \cup W_{U L} ; W_{U} \subset W$ :Set of unloading ODs $\left(W_{U}=\{1-5\}\right.$ for the system shown in Fig. 1$) ; W_{T F}$ : Set of oil transfer ODs from storage tank to charging tank $\left(W_{T F}=\{7,8,10,12-14\}\right.$ for the system shown in Fig. 1$)$; $W_{T O}$ : Set of oiltransfer ODs from charging tank to storage tank at site $\# 2\left(W_{T O}=\{6,9,11\}\right.$ for the system shown in Fig. 1$) ; W_{\mathrm{T}}=$ $W_{T F} \cup W_{T O} ; W_{T} \subset W$ : Set of oil transfer ODs $\left(W_{T}=\{6-14\}\right) ; W_{D} \subset W$ : Set of oil feeding ODs ( $W_{D}$ $=\{15-21\}) ; R_{V H} \subset R$ : Set of H-oil vessels at site \#2; $R_{V L} \subset R$ : Set of L-oil vessels at site \#1; $R_{V}=$ $R_{V H} \cup R_{V L} ; R=R_{V} \cup R_{S} \cup R_{C} \cup R_{D}$ : Set of resources; $\mathrm{r}_{\text {special }}$ :Storage tank at site \#2 which is used to eject the H-oil in pipeline2 after H-oil transfer finishing H-oil transportation; $\mathrm{r}_{\mathrm{v}}$ : Virtual storage tank for pipeline2; $R_{S H} \subset R$ : Set of H-oil storage tank at site \#2; $R_{S L} \subset R$ : Set of L-oil vessels at site \#1; $R_{S}=$ $R_{S H} \cup R_{S L} \cup\left\{r_{\text {special }}\right\} \cup\left\{r_{v}\right\} ; R_{S} \subset R$ : Storage tanks set; $R_{C} \subset R$ : Charging tanks set; $R_{S}=R_{S H} \cup R_{S L} ; R_{D} \subset$ $R$ : CDUs set; $I_{r} \subset W$ : Entrytransfer ODs set of resource $r$; $O_{r} \subset W$ : Exittransfer ODs set of resource $r$; $C$ : Set of crude oil types; $\mathrm{C}_{\mathrm{H}}$ : Set of H-oil types; $\mathrm{C}_{\mathrm{L}}$ : Set of L-oil types; $\mathrm{C}=C_{H} \cup C_{L}$; $H$ : Scheduling horizon; $\mathrm{V}_{\mathrm{p} 1}$ : Capacity of L-oil pipeline1; $\mathrm{V}_{\mathrm{p} 2}$ : Capacity of $\mathrm{H}$-oil pipeline2; $\mathrm{V}_{\text {hot }}$ : Indispensable hot L-oil volume which should run across Pipeline \#2 to heat it; RT: Oil residency time; $L V_{v}$ and $U V_{v}$ : Lower and upper bounds of the volume deliveredby OD $v$ and generally $L V_{v}=0$ for an OD except that an unloading OD is required to unload all the oil of a type in a vessel once, in this case, $L V_{v}=U V_{v}$; $L F_{v}$ and $U F_{v}$ are: Minimal and maximal flowrates permissive for OD $v, v \in W_{U} \cup W_{D} ; L F_{v h}$ and $U F_{v h}$ are: Minimal and maximal flowrates permissivefor Pipeline \#2; $L F_{v l}$ and $U F_{v l}$ are: Minimal and maximal flowrates permissive for Pipeline \#1; $S_{r}$ : Arrival time of vessel $r$; $F_{\text {pipeline2 }}$ : Transfer flow rate of Pipeline \#2; $F_{\text {pipeline1 }}$ : Transfer flow rate of Pipeline \#1; $L C_{r}$ and $U C_{r}$ : Capacity limit of tank $r \in R_{S} \cup R_{C} ; L D_{r}$ and $U D_{r}$ : Lower and upper bounds of demand on crude oil to be delivered from charging tank $r \in R_{C}$ during the scheduling horizon.

Variables:Binary assignment variables: $Z_{i v} \in\{0,1\}, i \in T$ and $v \in W$.If priority-slot $i c a n$ accommodateOD $v, Z_{i v}=1$, and otherwise $Z_{i v}=0$. Continuous time variables: $S_{i v} \geq 0$ and $D_{i v} \geq 0, i \in T$ and $v \in W$.If priority-slot ican accommodateOD $v, S_{i v}$ is the beginning time of OD $v$, andotherwise $S_{i v}=$ 0 . If priority-slot ican accommodateOD $v, D_{i v}$ is the duration ofOD $v$, andotherwise $D_{i v}=0$. Operation variables: $V_{i v} \geq 0$ and $V_{i v c} \geq 0, i \in T, v \in W$, and $c \in C$, where $V_{i v}$ is the entire volume of crude oil delivered by OD $v$ if priority-slot ican accommodateit, and otherwise $V_{i v}=0 . V_{i v c}$ is the crude oilvolume of type $c \in C$ delivered by OD $v$ if priority-slot $i c a n$ accommodateit, and otherwise $V_{i v c}=0$. Resource variables: $L_{i r}$ and $L_{i r c}, i \in T, r \in R$, and $c \in C$, where $L_{i r}$ is the entireaggregated volume of crude oil in $r \in R_{S} \cup R_{C}$ at the beginning of slot i. $L_{i r c}$ is the aggregated volume of crude oiltype $c$ in $r \in R_{S} \cup R_{C}$ at the beginning of slot $i$.

Auxiliary continuous variables: $\mathrm{x}>0$.

With the notation given above, we can present our formulation for the short-term crude oil operations scheduling problem with oil residency time constraints, high-fusion-point oil and two pipelines transfer being taken into account by using the priority-slot-based method. First, we present the constraints as follows.

\section{Common constraints:}

$$
\begin{aligned}
& \sum_{v \in W} Z_{i v}=1 \\
& S_{i v}+D_{i v} \leq H \times Z_{i v}, i \in T \text { and } v \in W \\
& V_{i v} \leq U V_{v} \times Z_{i v}, \quad i \in T \text { and } v \in W
\end{aligned}
$$


$V_{i v} \geq L V_{v} \times Z_{i v}, i \in T$ and $v \in W$

$\sum_{c \in C} V_{i v c}=V_{i v}, i \in T, v \in W$ and $c \in C$

$L_{i r}=L_{0 r}+\sum_{j \in T, j<i} \sum_{v \in I_{r}} V_{j v}-\sum_{j \in T, j<i} \sum_{v \in O_{r}} V_{j v}, i, j \in T, r \in R$

$L_{i r c}=L_{0 r c}+\sum_{j \in T, j<i} \sum_{v \in I_{r}} V_{j v c}-\sum_{j \in T, j<i} \sum_{v \in O_{r}} V_{j v c}, i, j \in T, r \in R$ and $c \in C$

$\sum_{i \in T} \sum_{v \in O_{r}} Z_{i v}=1, r \in R_{V}$

$\sum_{j \in T, j<i} \sum_{v \in O_{r 2}} Z_{j v}+\sum_{j \in T, j \geq i} \sum_{v \in O_{r 1}} Z_{j v} \leq 1, i \in T, i \neq 1$, and $r 1, r 2 \in R_{V H}$

$\sum_{j \in T, j<i} \sum_{v \in O_{r 2}} Z_{j v}+\sum_{j \in T, j \geq i} \sum_{v \in O_{r 1}} Z_{j v} \leq 1, i \in T, i \neq 1$, and $r 1, r 2 \in R_{V L}$

$\sum_{v \in W_{U H}}\left(S_{i v}+D_{i v}\right) \leq \sum_{v \in W_{U H}} S_{j v}+H \times\left(1-\sum_{v \in W_{U H}} Z_{j v}\right), i, j \in T$ and $i<j$

$\sum_{v \in W_{U L}}\left(S_{i v}+D_{i v}\right) \leq \sum_{v \in W_{U L}} S_{j v}+H \times\left(1-\sum_{v \in W_{U L}} Z_{j v}\right), i, j \in T$ and $i<j$

$\sum_{v \in I_{r}}\left(S_{i v}+D_{i v}\right) \leq \sum_{v \in O_{r}} S_{j v}+H \times\left(1-\sum_{v \in O_{r}} Z_{j v}\right), i, j \in T, i<j, r \in R_{S} \cup R_{C}$

$\sum_{v \in O_{r}}\left(S_{i v}+D_{i v}\right) \leq \sum_{v \in I_{r}} S_{j v}+H \times\left(1-\sum_{v \in I_{r}} Z_{j v}\right), i, j \in T, i<j, r \in R_{S} \bigcup R_{C}$

$\sum_{v \in O_{r}}\left(S_{i v}+D_{i v}\right) \leq \sum_{v \in O_{r}} S_{j v}+H \times\left(1-\sum_{v \in O_{r}} Z_{j v}\right), i, j \in T, i<j, r \in R_{S L} \bigcup R_{C}$

$\sum_{v \in O_{r}}\left(S_{i v}+D_{i v}\right)+V_{P 1} / F_{\text {pipeline } 1} \leq H, i \in T$ and $r \in R_{S L} \cup R_{C}$

$\sum_{v \in O_{r}}\left(S_{i v}+D_{i v}\right) \leq \sum_{v \in O_{r}} S_{j v}+H \times\left(1-\sum_{v \in O_{r}} Z_{j v}\right), i, j \in T, i<j, r \in R_{S H} \cup R_{C}$

$\sum_{v \in O_{r}}\left(S_{i v}+D_{i v}\right)+V_{P 2} / F_{\text {pipeline } 2} \leq H, i \in T$ and $r \in R_{S H} \cup R_{C}$

$\sum_{v \in I_{r}}\left(S_{i v}+D_{i v}\right) \leq \sum_{v \in I_{r}} S_{j v}+H \times\left(1-\sum_{v \in I_{r}} Z_{j v}\right), i, j \in T, i<j$ and $r \in R_{D}$

$S_{i v}+D_{i v} \leq S_{j v}+H \times\left(1-Z_{j v}\right), i, j \in T, i<j$ and $v \in W$

$\sum_{i \in T} \sum_{v \in I_{r}} D_{i v}=H, r \in R_{D}$

$S_{i v} \geq S_{r} \times Z_{i v}, i \in T, r \in R_{V}$ and $v \in O_{r}$

$L F_{v} \times D_{i v} \leq V_{i v} \leq U F_{v} \times D_{i v}, i \in T$ and $v \in W_{U} \bigcup W_{D}$

$L F_{v h} \leq F_{\text {pipeline } 2} \leq U F_{v h}$

$L F_{v l} \leq F_{\text {pipeline } 1} \leq U F_{v l}$

$\sum_{j \in T, j<i} \sum_{v \in O_{r}} V_{j v}-\sum_{r \in R_{C}} L_{i r}-V_{P} \geq \sum_{r \in R_{C}} \sum_{i \in T} \sum_{v \in O_{r}} V_{i v}, i=|T|$ and $r \in R_{S}$

$H$-oil continuous transfer and eject constraint: if $\min \left(S_{j v^{\prime}}-S_{i v}-D_{i v}\right)>0, Z_{k v^{\prime \prime}, v^{\prime \prime} \in O_{r}, r=r_{s p e c i a l}}=1$, $V_{i v^{\prime \prime}:}=V_{P 2}$, otherwise $Z_{k v^{\prime \prime}, v^{\prime \prime} \in O_{r}, r=r_{\text {special }}}=0$, indicating that two adjacent and closest H-oil transfers have no time interval and it need not eject the H-oil using L-oil.

$$
\left\{\begin{array}{l}
\min \left(S_{j v^{\prime}}-S_{i v}-D_{i v}\right) \geq \underset{\substack{v_{k v^{\prime \prime}} \\
v, v^{\prime} \in O_{r}, r \in R_{S H} \\
r=r_{\text {special }}}}{Z_{n^{\prime}}} \times x \\
Z_{k v^{\prime \prime}} \times V_{i v^{\prime \prime} c}=V_{P 2} \times Z_{k v^{\prime \prime}}
\end{array} \quad i, j, k \in T, i<k<j, c \in C, v, v^{\prime}, v^{\prime \prime} \in O_{r}, r \in R_{S H}\right.
$$

Heating pipeline2 and H-oil transfer beginning constraint:

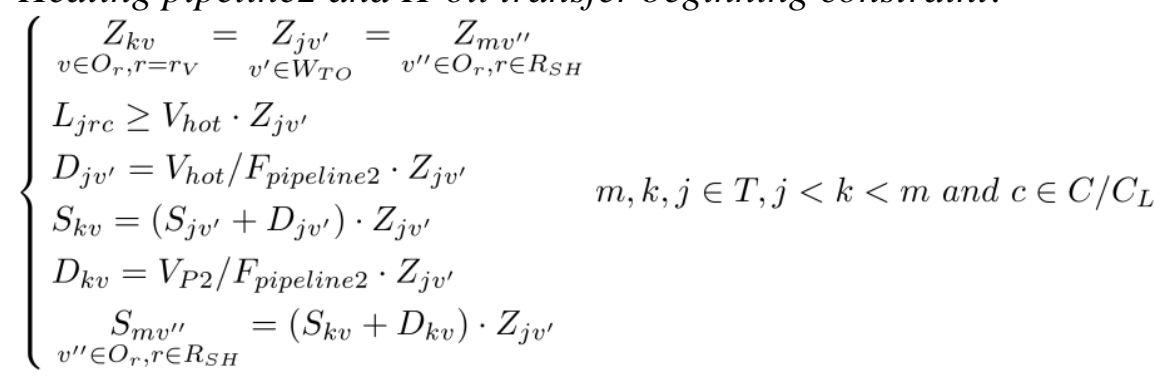

if $Z_{j v^{\prime} v^{\prime} \in W_{T O} O}, Z_{k v v \in O_{r}, r=r_{V}}=1$ and there is a charging tank transport hot L-oil to heat pipeline2 in preparation for H-oil transfer and $L_{j r c} \geq V_{h o t} \cdot Z_{j v^{\prime}}$ assures that its volume Ljrc is greater than Vhot, $D_{j v^{\prime}}=V_{h o t} / F_{\text {pipeline } 2}$. And then $Z_{k v, v \in O_{r}, r=r_{V}}=1$ compels the L-oil in rv to be ejected to some charging 
tank. H-oil begins to be transported at the same time $Z_{m v^{\prime \prime}, v^{\prime \prime} \in O_{r}, r \in R_{S H}}=S_{k v}+D_{k v}$. Otherwise, there is no L-oil reverse transfer, and $\mathrm{H}$-oil transfer will be impossible. Constraint (35) implies this process. Constraint (36) shows the volume of rspecial in time slot $i$.

$L_{i r c}=L_{0 r c}+\sum_{j \in T, j<i} \sum_{v \in I_{r}} V_{h o t}-\sum_{j \in T, j<i} \sum_{v \in I_{r}} V_{P 2}, i \in T, c \in C, r=r_{\text {special }}$

Objective function:The objective is to minimize the number of reverse transfer because a reverse transfer operation corresponds to an $\mathrm{H}$-oil setup and minimizing the number of reverse transfer means minimizing the total setup cost for $\mathrm{H}$-oil transportation. Therefore, the objective function is as follows.

$$
J=\sum_{i \in T} \sum_{v \in W_{T O}} Z_{i v}
$$

Based on the discussion above, the short-term crude oil operations scheduling problem with oil residency time constraints, high-fusion-point oil and two pipelines transfer can be formulated as the following mathematical programming problem.

Problem P1:Minimize $J=\sum_{i \in T} \sum_{v \in W_{T O}} Z_{i v}$

Subject to: constraints(1)-(36).

With the formulations for the scheduling problem developed above, we discuss how to solve the problem next.

\section{Solution Method}

As is presented in the last section, Problem P1 is indeed a mixed integer nonlinear programming (MINLP) model, because Constraint (34) and Constraint (35) have bilinear items. Thus, if the priority-slot number is known, we can use any generically NINLP solver such as DICOPT or BARONto solve the model. In fact, to obtain such a model, the key is to postulate on an appropriate priority slot number in advance. Unfortunately, it is rather difficult to predict the priority slotnumber. Therefore, it is of great necessity to develop an effective strategy to determine the priority slot number. To this ends, we present the following theorem before developing such a method.

Theorem 1: If $\mathrm{S}_{\mathrm{n}}$ is the optimal schedule obtained for Problem P1 with $\mathrm{n}$ being the number of priority slots, an optimal schedule $S_{k}$ for $P 1$ with $k>n$ is not better than $S_{n}$.

Proof: Without loss of generality, we assume that $S_{n}$ is formed by $n$ ODs $W_{i}, \in\{1,2, \ldots, n\}$, which are sequenced as $\mathrm{W}_{1} \mathrm{~W}_{2} \ldots \mathrm{W}_{\mathrm{n}}$ and the corresponding objective $\mathrm{J}=\mathrm{J}_{\mathrm{n}}$. Further, assume that, if the number priority slots is set to be $n+1$, there must be an OD inserted into sequence $W_{1} W_{2} \ldots W_{n}$, and a $\mathrm{n}+1$ ODs sequence $\mathrm{W}_{1}{ }_{1} \mathrm{~W}_{2}^{\prime}{ }_{2} \ldots \mathrm{W}_{\mathrm{n}}^{\prime} \mathrm{W}^{\prime}{ }_{\mathrm{n}+1}$ is formed. We assume that the inserted OD is $\mathrm{W}^{\prime}{ }_{\mathrm{j}}$. Then, there are three cases.

Case 1: $\mathrm{W}_{\mathrm{j}} \mathrm{W}_{\mathrm{U}}$, an oil unloading OD. It follows from objective $=\sum_{i \in T} \sum_{v \in W_{T}}+\sum_{i \in T} \sum_{v \in W_{D}}$ that $W_{j}^{\prime}$ has no effect on $J_{n+1}$, or we have $J_{n}=J_{n+1}$. This implies that $S_{n}$ and $S_{n+1}$ are equivalent in the sense of optimality.

Case 2: $\mathrm{W}^{\prime}{ }_{\mathrm{j}} \in \mathrm{W}_{\mathrm{T}}$, an oil transportation OD. In this case, the value of $\sum_{i \in T} \sum_{v \in W_{T}}$ in $\mathrm{J}$ is increased by one. At the same time, $\mathrm{W}_{\mathrm{j}} \in \mathrm{W}_{\mathrm{T}}$ has no effect on $\sum_{i \in T} \sum_{v \in W_{D}}$. Hence, we have $\mathrm{J}_{\mathrm{n}}+1=\mathrm{J}_{\mathrm{n}+1}$, or $\mathrm{S}_{\mathrm{n}}$ is better than $S_{n+1}$.

Case 3: $\mathrm{W}^{\prime}{ }_{\mathrm{j}} \in \mathrm{W}_{\mathrm{F}}$, an oil feeding OD. Similar to Case 2, it can be shown that $\mathrm{J}_{\mathrm{n}+1}=\mathrm{J}_{\mathrm{n}}+1$. In the same way, we can show that, $\forall \mathrm{i}>1$ and $\mathrm{k}=\mathrm{n}+\mathrm{i}$, we have $\mathrm{J}_{\mathrm{n}+\mathrm{i}} \geq \mathrm{J}_{\mathrm{n}}$, the theorem holds.

Theorem 1 shows that we can make a guess on the number of priority-slots in our model. Because of using SOS formulation, each operation executing exactly corresponds to only one priority-slot. It is rationally assumed that every operation executing once can make the system schedulable. With using SOS formulation, each operation executing corresponds to a priority-slot, therefore we can rationally select the number of all operations in a system as the priority-slot number $\mathrm{n}$ of our model, that is to say, $|\mathrm{T}|$ is $\mathrm{n}$. In fact, a smaller priority-slot number makes problem P1 more easily solved. Numerical experiment results show that our choice strategy for priority-slot number is reasonable. P1.

Based on Theorem 1, we propose optimization algorithm to find the best local solution of problem

Algorithm 1: Finding the best local optimal solution of problem P1 
Parameters t, result, and time can be set up and regulated according to the size of problem.

Step 1: Set $\mathrm{N}=\mathrm{n}, \mathrm{t}=30$ hours, results $=50$ and time $=0$ minutes;

Step 2: If time is less than $\mathrm{t}$, solve the problem P1 using DICOPT solver with priority-slot number $\mathrm{N}$, otherwise go to Step 6;

Step 3: If $\mathrm{P} 1$ is infeasible, let $\mathrm{N}=\mathrm{N}+1$, assign the solving time to time, go to Step 2;

Step 4: get the local optimal solution; if the value of local optimal solution is less than result, assign its value to result; assign the solving time to time, let $\mathrm{N}=\mathrm{N}+1$, go to Step 2;

Step 5: find the best local optimal solution of problem P1, go to Step 7;

Step 6: problem P1 cannot solve in expected time;

Step 7: calculations stop.

In actual use of Algorithm 1, we can arbitrarily choose a priority-slot number. If we cannot find the local optimal solution using the number, a greater number should be used to solve the problem until the solver cannot finds more perfect solution or computing time exceeds the upper bound of given time.

\section{Conclusion}

Thiswork presentsa more academically challenging system than the previous one-pipelineone. A new objective is introduced thatmaximizing the $\mathrm{H}$-oil volume on the basis of any individualH-oil transfer setup. It uses mathematical programming method to obtain a feasible schedule which transports as much $\mathrm{H}$-oil as possible andto minimize the setup cost of $\mathrm{H}$-oil. This model is MINLP model, so it cannot be solved effectively by CPLEX.We have to use the generic solver DICOPT to solve this model.We propose Algorithm 1 using DICOPTsolver. It reveals thatthe system's initialstate has great effect on theH-oil amountthat can be transferred,especially the flow rate of pipeline2. Therefore, before starting H-oil transfer we must design a plan of the initial conditions.

Considering optimization objectives else to satisfy diverse industrial demandsis our future work.On the other hand, when our model is huge, how to solve it using commercial software combined with branch-and-price and column generation also is our important future issue.

\section{Funding}

This work was partly supported by Natural Science Foundation of Guangdong Province under grants2014A030310288.

\section{References}

[1] C. A. Floudas and X. Lin, "Continuous-time versus discrete-time approaches for scheduling of chemical processes: A review," Computers \& Chemical Engineering, vol. 28, 2109-2129, 2004.

[2] C. A. Mendez and J. Cerda, "Dynamic scheduling in multiproduct batch plants," Computers \& Chemical Engineering, vol. 27, pp. 1247-1259, 2003.

[3] N. Q. Wu, M. C. Zhou, L. P. Bai, and Z. W. Li. "Short-term scheduling of crude oil operations in refinery withhigh-fusion-point oil and two transfer pipelines. " Enterprise Information Systems, in press, 2014.

[4] S. Mouret, I. Grossmann, and P. Pestiaux, "A novel priority-slot based continuous-time formulation for crude-oil scheduling problems," Industrial and Engineering Chemistry Research, vol. 48, no. 18, 8515-8528, 2009. 